\title{
Acción del Etanol sobre el Epitelio Nasal y Glándulas Septales de Ratas, Durante la Lactancia
}

\author{
Action of Ethanol on Rat Pups Nasal Epithelium and Septal Glands, During Lactation \\ "Marcos Marcondes de Godoy; ".** Ruberval A. Lopes; *"Miguel A. Sala; "Dionísio Vinha \& *"Simone C. H. Regalo
}

GODOY, M. M.; LOPES, R. A.; SALA, M. A.; VINHA, D. \& REGALO, S. C. H. Acción del etanol sobre el epitelio nasal y glándulas septales de ratas, durante la lactancia. Int. J. Morphol., 23(4):293-300, 2005.

RESUMEN: El consumo materno de etanol durante la lactancia altera la composición de la leche, provoca la aparición de etanol y acetaldehído en la leche y agrava los efectos del etanol en las crías de ratas. De este modo, fueron estudiados los efectos del etanol, administrado a las ratas madres durante la lactancia, sobre el epitelio respiratorio y en las glándulas septales anterior y posterior de las crías lactantes con 21 días de vida postnatal. Las ratas recibieron etanol al 20\% en el bebedero, ad libitum durante los 21 días que amamantaron. Los controles recibieron un volumen similar de agua sin alcohol. Las crías fueron sacrificadas en el $21^{\circ}$ día. Las cabezas fueran cortadas frontalmente. Los cortes seriados de $6 \mu \mathrm{m}$ de grosor fueron teñidos con hematoxilina y eosina. Fueron determinados los parámetros nucleares de los epitelios respiratorio y glandular, así como los volúmenes citoplasmático y celular, relación núcleo-citoplasma, densidades numérica y superficial y espesor del epitelio. El peso corporal medio de las crías control fue 34,9 g y en las tratadas 20,2 g. Histologicamente, el epitelio respiratorio se mostró adelgazado en el grupo tratado, constituido por células abundantes y menores, con núcleos menores. La glándula septal posterior presentó ácinos menores. En este trabajo, el etanol indujo un cuadro de hipotrofia epitelial (respiratorio y glandular), indicando una acción directa sobre las células de la mucosa nasal, además de retardar el desarrollo de las crías intoxicadas.

PALABRAS CLAVE: Alcoholismo; Cariometría; Estereología; Epitelio Respiratorio, Glándulas Septales; Lactancia; Rata.

\section{INTRODUCCIÓN}

El alcoholismo es un problema de salud pública que afecta millones de personas de toda edad, asociado a crímenes violentos y accidentes de tránsito con muertes o lesiones graves. Aristóteles ya observó que mujeres dipsómanas daban a luz bebés, como ellas, taciturnos e indolentes. Los efectos teratogénicos del etilismo materno sólo se aceptaron después de los trabajos de Le Moine et al. (1968) y Jones et al. (1973). El etilismo materno se asocia al "síndrome de alcoholismo fetal", que incluye restricción de crecimientos prenatal y postnatal y malformaciones múltiples (Abel, 1984).

Concentraciones altas de etanol, administradas como parte de dieta líquida o en el agua de beber, reducen notablemente el consumo de alimentos, causando malnutrición en mujeres grávidas o que amamantan (Shorey et al., 1977). La administración de dieta líquida, con 35\% de las calorías en forma de etanol, a ratas grávidas o lactantes, reduce el crecimiento de las crías más que la privación nutricional (Detering et al., 1979). El consumo materno crónico de alcohol durante la lactacia causa importantes disturbios metabólicos y alteración funcional de la glándula mamaria, que reduce la producción láctea, aumenta su tenor en gorduras, disminuye el contenido proteico y comienza a excretar etanol y acetaldehído, agravando los efectos neonatales del etanol (Guerri \& Sanchis, 1986; Vilaró et al., 1987; Tavares-doCarmo et al., 1996). La severa malnutrición que sufren las crías por la disminución de producción láctea, es compensada, en parte, por el aumento del tenor lipídico de la leche, induciendo adaptaciones metabólicas para prevenir la hipoglucemia grave y mantener los niveles mínimos de glucógeno hepático, pero, esas adaptaciones son insuficientes para permitir el desarrollo normal del cerebro, disminuido en esos animales (Tavares-do-Carmo et al., 1999).

\footnotetext{
* Universidade de Franca, Brasil.

** Departamento de Morfologia, Estomatologia e Fisiologia da Faculdade de Odontologia de Ribeirão Preto da Universidade de São Paulo, Brasil.

Financiado por la Universidad de Franca (UNIFRAN), Brasil.

Disertación de Maestria del primer autor, presentada a la Universidad de Franca (Área de Concentración en Promoción de Salud).
} 
Los niveles plasmáticos de prolactina y oxitocina post-succión son inhibidos por la acción del etanol (Fuchs, 1969; Coiro et al., 1992; Volpi et al., 1994). Ratas lactantes sometidas al etanol y separadas de las crías no varían los niveles de prolactina basal, mientras que las que continuan amamantando sufren inhibición de la prolactina (Subramanian \& Abel, 1988). La inhibición de la eyección láctea por alcohol es dosis-dependiente en la mujer (Cobo, 1973). La administración de etanol durante los períodos de 5 a 8 y de 9 a 12 días de lactancia inhibe la liberación de prolactina estimulada por la succión y disminuye el consumo de leche por las crías (Subramanian, 1997).

En seres humanos, el etanol ingerido en la leche materna causa efectos leves pero significativos en el desarrollo motor de lactantes (Little et al., 1989). Es común recomendar el consumo de pequeñas dosis de alcohol como factor auxiliar en la lactancia, ya que aporta calorías y fluidos adicionales y facilita el reflejo de eyección (Auerbach et al., 1987; Menella \& Beauchamp, 1993). El consumo seguro de alcohol, en relación a la ausencia de efectos nocivos, parece depender de la dosis ingerida. En ratas lactantes, aún dosis pequeñas $(5 \%)$, reducen el consumo de alimentos y la concentración de retinol en la leche (Albuquerque et al., 1998).

Fue sugerido que la inhibición de la oxitocina, y no la prolactina (estimulada por la succión), sería responsable de la restricción de crecimiento de las crías lactantes de ratas tratadas con etanol (Heil \& Subramanian, 2000). El consumo materno de alcohol afecta el desarrollo de las crías en forma dosis-dependiente. Así, la ingestión de altas dosis (10\% a 20\%) en el agua del bebedero causa reducción de los pesos corporal y hepático y altera el metabolismo de la glucosa cerebral, mientras que la ingestión de dosis baja de etanol (5\%) no altera los pesos corporal, cerebral y hepático de las crías, pero afecta el metabolismo cerebral de la glucosa (Oyama et al., 2000 a,b).

Considerando que la lactancia es un período importante y vulnerable, durante el cual la mayoría de los órganos aún está en desarrollo, y que la exposición materna al alcohol en esta fase podría causar efectos adversos en las crías, son objetivos de este trabajo estudiar histopatológicamente e histométricamente el epitelio respiratorio nasal y las glándulas septales anterior y posterior.

\section{MATERIAL Y MÉTODO}

Fueron usadas 10 ratas Wistar preñadas, con peso medio de $200 \mathrm{~g}$, alojadas en jaulas individuales y alimentadas con dieta comercial (Purina) y agua ad libitum.
En el día del parto (día 1) las crías fueron reducidas a 10 por madre. Fue administrado etanol al 20\%en el agua del bebedero, durante 21 días (lactancia). Al fin del $21^{\circ}$ día, las crías fueron sacrificadas mediante inyección de Hypnol® al $3 \%$. Las cabezas fueron separadas de los cuerpos y fijadas en solución de alcohol $80 \%$ - $85 \mathrm{ml}$, formol puro - $10 \mathrm{ml} \mathrm{y}$ ácido acético - $5 \mathrm{ml}$, por $24 \mathrm{~h}$, a temperatura ambiente. Después de descalcificadas en solución a:a de citrato de sodio al $20 \%$ y ácido fórmico al 30\%, las cabezas fueron desarticuladas y seccionadas frontalmente a nivel de los molares. Cortes seriados, de $6 \mu \mathrm{m}$ de grosor, fueron teñidos con hematoxilina y eosina.

Se estimaron los siguientes parámetros cariométricos (Sala et al., 1994): diámetro medio, relación diámetro mayor/diámetro menor, perímetro, área, volumen, relación volumen/área, coeficiente de forma, índice de contorno y excentricidad.

Los siguientes parámetros estereológicos fueron estimados en el epitelio respiratorio: relación núcleo-citoplasma, volúmenes citoplasmático y celular, densidad numérica, relación superficie externa/superficie basal y espesor medio epitelial (Sala et al., 1992). En las glándulas septales fueron estimados: diámetro y espesor medio de los acinos y conductos (Sala et al., 1981) y densidad de superficie (Tomkeieff, 1945).

El análisis estadístico fue efectuado mediante la prueba no paramétrica de Wilcoxon-Mann-Whitney (Sprent \& Smeeton, 2001).

\section{RESULTADOS}

El peso de las crías de ratas tratadas con etanol durante la lactancia, es significativamente menor $(20,20 \mathrm{~g})$ que el de las crías control $(34,86 \mathrm{~g})(\mathrm{p}<0,01)$.

El epitelio respiratorio en los animales control es de tipo seudoestratificado cilíndrico ciliado, constituido por 6 tipos celulares: células cilíndricas ciliadas y no ciliadas, células caliciformes, células en cepillo, células cuboides y células basales (Fig. 1). La lámina propia está constituida por tejido conjuntivo laxo altamente vascularizado, con numerosas glándulas mucosas y serosas (Fig. 1). Las glándulas septales anteriores son de tipo tubuloacinar, constituidas por numerosos acinos solitarios y un largo conducto único que desemboca en el vestíbulo. La secreción glandular es principalmente serosa. Las glándulas septales posteriores son semejantes a las anteriores y su secreción es de naturaleza mucosa (Figs. 3 y 5 ). 
El epitelio respiratorio en las crías de ratas tratadas con etanol es más delgado, constituido por células menores con menos citoplasma y núcleos menores. El número de células por $\mathrm{mm}^{3}$ está aumentado (Fig. 2).

La glándula septal anterior no muestra diferencia significativa en tamaño, pero los núcleos de las células de los acinos son menores (Fig. 4). La glándula septal posterior presenta acinos menores con núcleos también menores (Fig. 6).
Los parámetros cariométricos de las células cilíndricas ciliadas del epitelio respiratorio revelan núcleos significativamente menores en el grupo tratado, mientras que la forma nuclear es semejante en ambos grupos (Tabla I).

Los núcleos de las células de los acinos de las glándulas septales anteriores y posteriores son menores en la rata tratada y muestran alteración de su forma. Los núcleos de las células de los conductos presentan tamaño similar, pero con alteración de forma (Tabla I).
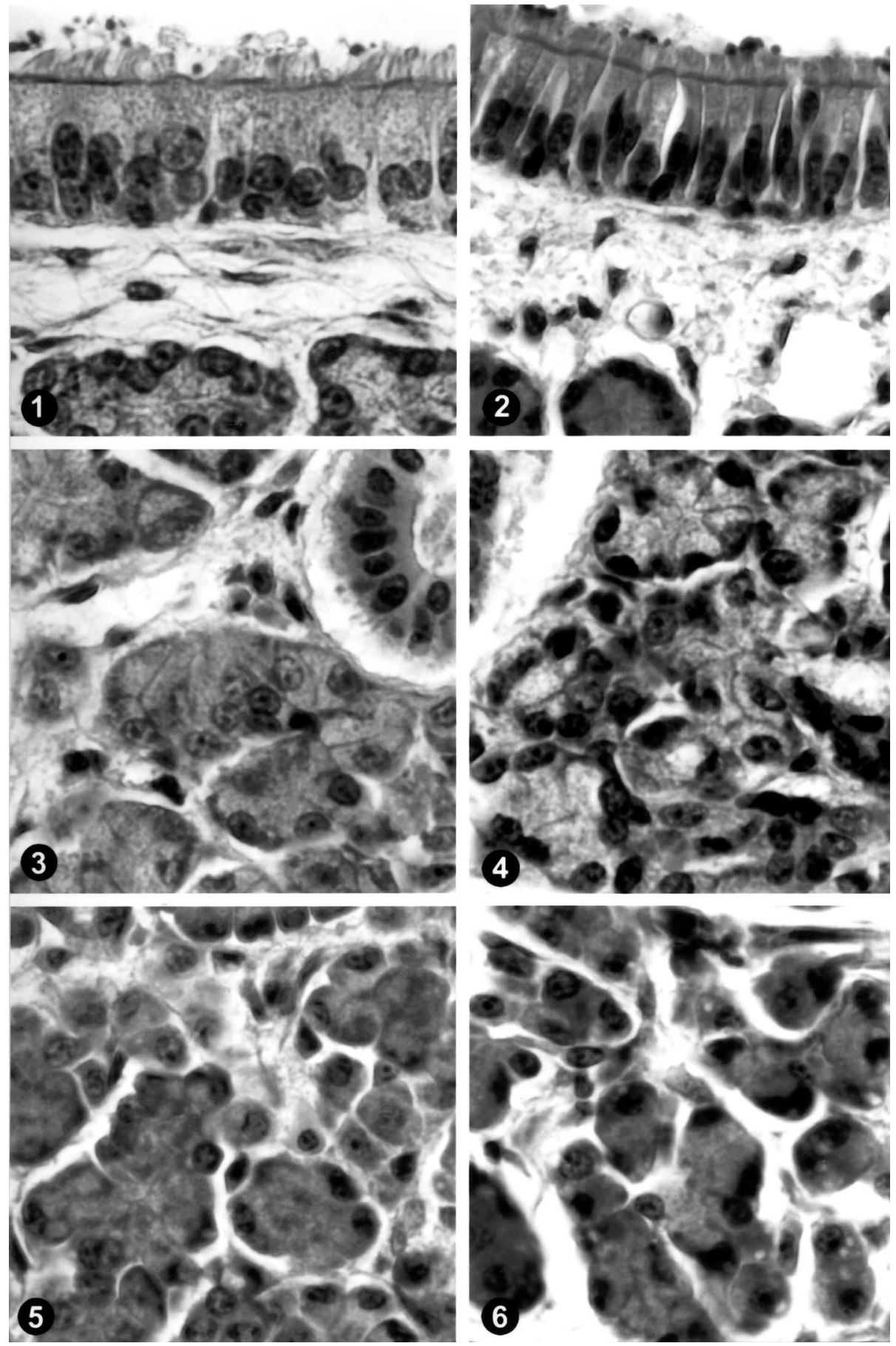

Los volúmenes citoplasmático y celular y el espesor del epitelio respiratorio son significativamente menores, mientras que la relación núcleo-citoplasma, densidad de superficie y densidad numérica son mayores (Tabla II). En los acinos de la glándula septal posterior el diámetro medio y el grosor de la pared son menores y la densidad de superficie es mayor en las ratas tratadas. No se observaron alteraciones en la glándula septal anterior (Tabla III).

Fig. 1. Aspecto histológico del epitelio respiratorio de cría de rata control. Observar las células cilíndricas ciliadas, lámina propia y glándula septal. HE $(900$ x).

Fig. 2. Aspecto histológico del epitelio respiratorio de cría de rata tratada. Observar las alteraciones evidentes en las células ciliadas, lámina propia con tejido conjuntivo desorganizado y acinos glandulares. HE (900 x).

Fig. 3. Aspecto histológico de la glándula septal anterior de cría de rata control. Observar acinos, conductos y tabiques de tejido conjuntivo. HE (900 x).

Fig. 4. Aspecto histológico de la glándula septal anterior de cría de rata tratada. Observar acinos menores y núcleos de volumen menor. HE (900 x).

Fig. 5. Aspecto histológico de la glándula septal posterior de cría de rata control. Observar acinos bien constituidos. HE (900 x).

Fig. 6. Aspecto histológico de la glándula septal posterior de cría de rata tratada. Observar acinos menores, con núcleos también menores. HE (900 x). 
Tabla I. Valores medios de los parámetros cariométricos del epitelio respiratorio y de las glándulas septales de ratas control y sometidas a los efectos del etanol durante la lactancia. Prueba de Wilcoxon-Mann-Whitney.

\begin{tabular}{|c|c|c|c|c|c|c|c|c|c|c|}
\hline \multirow{3}{*}{ Parámetros } & \multirow{2}{*}{\multicolumn{2}{|c|}{ Epitelio respiratorio }} & \multicolumn{4}{|c|}{ Glándula septal anterior } & \multicolumn{4}{|c|}{ Glándula septal posterior } \\
\hline & & & \multicolumn{2}{|c|}{ Acinos } & \multicolumn{2}{|c|}{ Conductos } & \multicolumn{2}{|c|}{ Acinos } & \multicolumn{2}{|c|}{ Conductos } \\
\hline & $\mathrm{C}$ & $\mathrm{T}$ & $\mathrm{C}$ & $\mathrm{T}$ & $\mathrm{C}$ & $\mathrm{T}$ & $\mathrm{C}$ & $\mathrm{T}$ & $\mathrm{C}$ & $\mathrm{T}$ \\
\hline Diámetro mayor $(\mu \mathrm{m})$ & 7,36 & $5,30^{* *}$ & 5,56 & $5,19 *$ & 5,44 & 5,26 & 5,43 & 5,06 & 5,42 & 5,12 \\
\hline Diámetro menor ( $\mu \mathrm{m})$ & 5,58 & $3,99 * *$ & 3,80 & $3,05 * *$ & 3,57 & 3,10 & 4,17 & $3,52 * *$ & 3,96 & 3,65 \\
\hline Diámetro medio $(\mu \mathrm{m})$ & 6,39 & $4,59^{* *}$ & 4,58 & $3,95^{* *}$ & 4,39 & 4,01 & 4,75 & $4,21^{*}$ & 4,62 & 4,39 \\
\hline Relación D/d & 1,33 & 1,33 & 1,50 & $1,79 * *$ & 1,56 & $1,77^{* *}$ & 1,33 & $1,48^{* *}$ & 1,39 & $1,50^{*}$ \\
\hline Perímetro $(\mu \mathrm{m})$ & 20,44 & $14,69 * *$ & 14,86 & $13,20 * *$ & 14,33 & 13,78 & 15,16 & $13,62^{*}$ & 14,84 & 14,23 \\
\hline Área $\left(\mu \mathrm{m}^{2}\right)$ & 32,49 & $16,76^{* *}$ & 16,71 & $12,53^{* *}$ & 15,43 & 12,92 & 17,98 & $14,25^{*}$ & 17,16 & 15,46 \\
\hline Volumen $\left(\mu \mathrm{m}^{3}\right)$ & 142,02 & $52,63 * *$ & 52,49 & $34,38^{* *}$ & 47,03 & 36,19 & 58,64 & $41,90^{*}$ & 55,38 & 47,23 \\
\hline Relación V/A & 4,26 & $3,06^{* *}$ & 3,05 & $2,63 * *$ & 2,92 & 2,68 & 3,17 & $2,80^{*}$ & 3,08 & 2,92 \\
\hline Excentricidad & 0,63 & 0,63 & 0,71 & $0,79 * *$ & 0,73 & $0,79 * *$ & 0,62 & $0,70 * *$ & 0,66 & $0,71 *$ \\
\hline Coeficiente de forma & 0,96 & 0,96 & 0,94 & $0,88 * *$ & 0,93 & $0,89 * *$ & 0,97 & $0,94 * *$ & 0,96 & $0,94 *$ \\
\hline Índice de contorno & 3,61 & 3,62 & 3,67 & $3,79 * *$ & 3,69 & $3,78^{* *}$ & 3,61 & $3,66^{* *}$ & 3,63 & $3,67^{*}$ \\
\hline
\end{tabular}

$(*) \mathrm{p}<0,05 \quad(* *) \mathrm{p}<0,01$

Tabla II. Valores medios de los parámetros estereológicos del epitelio respiratorio de ratas controles y sometidas a los efectos del etanol durante la lactancia. Prueba de Wilcoxon-Mann-Whitney.

\begin{tabular}{lrc}
\hline \multicolumn{1}{c}{ Parámetros } & Control & Tratado \\
\hline Volumen citoplasmático $\left(\mu \mathrm{m}^{3}\right)$ & 474,82 & $48,73^{*}$ \\
Volumen celular $\left(\mu \mathrm{m}^{3}\right)$ & 610,53 & $99,60^{*}$ \\
Relación N/C & 0,11 & $0,21^{*}$ \\
Densidad de superficie $\left(\mathrm{mm}^{2} / \mathrm{mm}^{3}\right)$ & 17,42 & $24,04^{*}$ \\
Grosor total $(\mu \mathrm{m})$ & 78,09 & $55,31^{*}$ \\
Longitud de los cilios $(\mu \mathrm{m})$ & 17,93 & 11,27 \\
Relación sup. externa/basal & 0,96 & 0,99 \\
Densidad numérica $\left(\mathrm{n} / \mathrm{mm}^{3} \times 10^{6}\right)$ & 1,76 & $10,24^{*}$ \\
\hline
\end{tabular}

(*) $\mathrm{p}<0,01$

Tabla III. Valores medios de los parámetros estereológicos de los acinos de las glándulas septales de ratas controles (C) y sometidas a los efectos del etanol durante la lactancia (T). Prueba de Wilcoxon-Mann-Whitney.

\begin{tabular}{lccrr}
\hline \multirow{2}{*}{ Parámetros } & \multicolumn{2}{c}{$\begin{array}{c}\text { Glándula septal } \\
\text { anterior }\end{array}$} & $\mathrm{T}$ & \multicolumn{2}{c}{$\begin{array}{c}\text { Glándula septal } \\
\text { josterior }\end{array}$} \\
\cline { 2 - 5 } & $\mathrm{C}$ & $\mathrm{C}$ & $\mathrm{T}$ \\
\hline Densidad volumétrica $(\%)$ & 0,3850 & 0,3570 & 0,4400 & 0,4500 \\
Densidad de superficie $\left(\mathrm{mm}^{2} / \mathrm{mm}^{3}\right)$ & 231,16 & 190,14 & 213,94 & $297,54^{* *}$ \\
Diámetro medio $(\mu \mathrm{m})$ & 68,3 & 75,14 & 76,78 & $60,28^{*}$ \\
Grosor de la pared $(\mu \mathrm{m})$ & 32,46 & 35,50 & 38,36 & $30,12^{*}$ \\
\hline
\end{tabular}

${ }^{(*)} \mathrm{p}<0,05(* *) \mathrm{p}<0,01$

\section{DISCUSIÓN}

Los pesos medios de las crías de este trabajo, fueron $34,86 \mathrm{~g}$ para los controles y $20,2 \mathrm{~g}$ para los tratados, siendo esta diferencia significativa.

Abel (1974) observó que las crías de madres que bebían etanol, presentan menor crecimiento que los controles. En ratas, después del destete, el etanol causa reducción de peso corporal (Wallgren et al., 1967). En lauchas que amamantan, el etanol inyectado retarda el crecimiento de las crías, pero en el bebedero no altera su crecimiento (Abel, 1974). Según Oyama et al. (2000a, b) la ingestión materna de etanol afecta el desarrollo de las crías en forma dosis-dependiente: al $10 \%$ o $20 \%$ reduce el peso corporal de las crías, mientras que al $5 \%$ no modifica el peso de las mismas.
Se sabe que el alcoholismo durante el embarazo provoca severa deficiencia del crecimiento prenatal y estudios experimentales muestran bajo peso al nacer en crías de ratas tratadas con alcohol (Detering et al.; Ludeña et al., 1983; Testar et al., 1988). El tratamiento crónico con etanol causa disturbios metabólicos maternos durante la lactancia, alterando la función de la glándula mamaria, que disminuye la producción láctea y aumenta los lípidos (Vilaró et al., 1987).

El epitelio respiratorio de las crías de ratas tratadas con etanol al $20 \%$ en el agua del bebedero, se mostró más delgado, constituido por células menores con citoplasma escaso y núcleos menores. La morfometría confirmó esas observaciones: el espesor del epitelio fue menor, consti- 
tuido por células menores y más numerosas. El núcleo de la célula cilíndrica era menor y no presentó alteración de su forma.

La glándula septal anterior no mostró diferencias en tamaño, pero el núcleo de las células de los acinos era menor. Los gránulos de secreción eran escasos, causando disminución de la basofilia citoplasmática. La estereología confirmó estas observaciones, sin diferencias significativas entre los dos grupos. El núcleo de las células de los ácinos era menor, con forma más alargada en los tratados. El núcleo de las células de los conductos glandulares no mostró diferencia de tamaño, pero su forma era más alargada en los tratados.

La glándula septal posterior mostró acinos menores, con núcleos menores. La estereología demostró que el núcleo de las células de los acinos era menor, con forma más alargada en los tratados. El núcleo de los conductos era semejante en los dos grupos.

Resumiendo, las modificaciones encontradas en el epitelio respiratorio y en las glándulas septales evidencian un cuadro de hipotrofia celular.

Estas alteraciones pueden ser provocadas por los dos mecanismos de acción del etanol: directa sobre los tejidos e indirecta, por la desnutrición causada por el alcohol. Fue demostrado que el etanol, su metabolito acetaldehído alteran la síntesis proteica, inhibiendo su transporte y secreción en el hígado (Baraona \& Lieber, 1982). Ambos compuestos alteran in vivo o in vitro los microtúbulos (Matsuda et al., 1979; Baraona et al., 1984), causando acumulación intracelular de vesículas secretoras y dilatación generalizada de las cisternas del retículo endoplasmático (Feldman \& Maurice, 1977).

El consumo crónico de etanol altera profundamente la función de la glándula mamaria, con disminución de producción de leche, modificación de su composición y de la estructura de la glándula mamaria (Vilaró et al., 1987), con alteración de la producción de aminoácidos (Viñas et al., 1987). Además, se demostró la presencia de etanol y acetaldehído en la leche de ratas tratadas crónicamente con etanol. Luego de ingerido, el etanol pasa hacia la leche, donde puede ser encontrado en concentración similar a la de la sangre, debido a la ausencia alcohol-deshidrogenasa en la glándula mamaria (Guerri \& Sanchis; Vilaró et al., 1987). Al contrario, la concentración de acetaldehído en la leche es menor que en la sangre, dada la alta actividad en acetaldehído-deshidrogenasa de la glándula mamaria, aúnque disminuida por el consumo prolongado de etanol (Guerri \& Sanchis). La pérdida de polarización celular de la glándula mamaria, con reducción de los dictiosomas del aparato de Golgi y anomalías severas de la maduración y secreción de caseína, sin cambios en la síntesis y secreción de lípidos, fue atribuida a la acción directa del etanol y/o del acetaldehído sobre la glándula mamaria (Vilaró et al., 1989).

A pesar que 1 gramo de etanol produce $29,7 \mathrm{~kJ}$, el consumo de alcohol puede inducir desnutrición por 3 vías diferentes: 1) reducción de ingestión de alimentos; 2) disminución de la digestión y absorción de alimentos; y 3) alteración del metabolismo del nutriente (Lieber, 1983; Testar et al., 1986).

El aumento de la ingestión de alimentos que ocurre en las madres control con la lactancia, es pequeño y temporal en las madres tratadas con etanol, causando marcada pérdida de peso durante los primeros 15 días de lactancia. En estos animales, la secreción láctea está reducida, como ocurre con las mujeres que consumen alcohol cuando amamantan (Cobo; Rosett, 1979). La falta de leche disponible en ratas lactantes es la causa principal de la severa desnutrición observada por Ludeña et al., en crías de 15 días y manifestada por restricción de crecimiento y pérdida de las reservas de glucógeno hepático. La concentración de cuerpos cetónicos en la sangre de estas crías está levemente aumentada, debido al aumento de la degradación de ácidos grasos causado por la desnutrición. Esta condición es similar a la causada por la inanición prolongada en ratas adultas (Herrera \& Freinkel, 1968), donde los niveles de cuerpos cetónicos circulantes están bajos, en comparación a los animales sometidos a períodos cortos de privación de alimentos, debido a la falta de reservas lipídicas a ser movilizadas y oxidadas. Esta explicación puede ser aplicada también a las crías de madres alcoholizadas, en las cuales, junto con la falta de reservas lípídicas, hay una reducción drástica en la leche disponible, fuente principal de gorduras en la rata que amamanta (Aranda et al., 1973).

La ingestión continua de alcohol durante la lactancia modifica la composición química de la leche, con aparición de etanol y acetaldehído e intensifica los efectos del etanol en los neonatos (Guerri et al., 1984; Sanchis \& Guerri, 1986; Viñas et al.). En ratas que recibieron etanol en el agua del bebedero, las glándulas mamarias muestran disminución de peso y alteraciones histológicas en la gordura y células epiteliales (Jones \& Stewart, 1984). Esas alteraciones estructurales pueden contribuir a los defectos funcionales observados en las glándulas mamarias de los animales tratados con etanol.

Usando ratas alimentadas con dieta pareada conteniendo etanol o dieta control líquida isocalórica o ración 
comercial, se demostró disminución del epitelio alveolar y aumento del tejido conjuntivo en el $2^{\circ}$ día de lactancia de animales tratados con etanol por tiempo prolongado, comparados con controles-pareados y alimentados con ración comercial (Steven et al., 1989). Entre tanto, al 10º́a de la lactancia no ocurrieron alteraciones de la estructura glandular. Estos resultados indican que el etanol altera la estructura de la glándula mamaria durante los primeros estados de la lactancia, aunque existan niveles adecuados de proteína en la dieta.

Los resultados de este trabajo sugieren que las crías de madres tratadas con etanol al 20\%, durante la lactancia, presentan retardo del crecimiento postnatal, con epitelio respiratorio formado por células hipotróficas y con glándulas septales posteriores reducidas y también hipotróficas.

GODOY, M. M.; LOPES, R. A.; SALA, M. A.; VINHA, D. \& REGALO, S. C. H. Action of ethanol on rat pups nasal epithelium and septal glands, during lactation. Int. J. Morphol., 23(4):293-300, 2005.

SUMMARY: Maternal ingestion of ethanol during lactation alters the chemical composition of milk, results in the appearance of ethanol and acetaldehyde in milk, and exacerbates the effects of ethanol on the rat pups. So that, the effect on respiratory epithelium and anterior and posterior septal glands in 21-day-old suckling pups of ethanol treated mothers was studied. Female rats received dinking water ad libitum containing ethanol (20\%) throughout the whole lactation. Control animals received a similar volume of water without ethanol. Lactent rats (21 day-old) were killed by lethal dose of anesthetic. The heads were serially sectioned ( $6 \mu \mathrm{m}$ thick) in a frontal plain, and stained with haematoxylin and eosin. Cell nuclear parameters were estimated, as well as cytoplasm and cell volume, nucleuscytoplasm ratio, number and surface densities, and epithelial thickness. Mean body weight of the pups was $34.9 \mathrm{~g}$ for the controls and $20.2 \mathrm{~g}$ for the treated group. Histologically, the respiratory epithelium was thinner, with more numerous and smaller cells and small nuclei. The posterior septal glands showed smaller acini. In this paper, ethanol induced epithelial (respiratory and glandular) hypotrophy, indicating a direct action in nasal mucous cells, besides retarded development in pups.

KEY WORDS: Alcohol; Karyometry; Stereology; Respiratory Epithelium; Septal Glads; Lactation; Rat.

\section{REFERENCIAS BIBLIOGRÁFICAS}

Abel, E. L. Alcohol ingestion in lactating rats: effects on mother and offspring. Arch. Int. Pharmacodyn., 210: 121$7,1974$.

Abel, E. L. Fetal alcohol syndrome and fetal alcohol effects. New York, Plenum Press, 1984. pp.183-205.

Albuquerque, K. T.; Ramalho, R. A.; Soares, A G. \& Tavaresdo-Carmo, M. G. Effects of ethanol intake on retinol concentration in the milk of lactating rats. Braz. J. Med. Biol. Res., 31:929-32, 1998.

Aranda, A.; Blazquez, E. \& Herrera, E. Liver components, blood glucose and ketone bodies in fed and starved suckling rats. Horm. Metab. Res., 5:350-5, 1973.

Auerbach, K. G.; Blume, S.; Schreiber, J. R. \& Falkner, F. Beer and the breast-feeding mom. J. Am. Med. Assoc., 258:2126, 1987.

Baraona, E. \& Lieber, C.S. Effects of ethanol on hepatic transport of proteins. Ann. Rev. Med., 33:281-92, 1982.

Baraona, E.; Finkelman, F. \& Lieber, C. S. Reevaluation of the effects of alcohol consumption on rat liver microtubules. Res. Comm. Chem. Pathol. Pharmacol., 44:265-78, 1984.

Cobo, E. Effect of different doses of ethanol on the milk ejecting reflex in lactating women. Am. J. Obstet. Gynecol., 115:817-21, 1973.

Coiro, V.; Alboni, A.; Gramellini, D.; Cigarini, C.; Bianconi, L.; Pignatti, D.; Volpi R. \& Chiodera, P. Inhibition by ethanol of the oxytocin response to breast stimulation in normal women and the role of endogenous opioids. Acta Endocrinol., 126:213-6, 1992.

Detering, N.; Reed, W. D.; Ozand, P. T. \& Karahasan, A. The effects of maternal ethanol consumption in the rat on the development of their offspring. J. Nutr., 109: 9991009, 1979.

Feldman, G. \& Maurice, M. Morphological findings of liver protein synthesis and secretion. In: Hopper, H.; Bianchi L. \& Reutter W. (eds). Membrane Alterations as Basis of Liver Injury. Lancaster. English Medical \& Technical Publ., 1977. pp. 61-76. 
Fuchs, A R. Ethanol and inhibition of oxytocin release in lactating rats. Acta Endocrinol., 62:546-54, 1969.

Guerri, C.; Esquifino, A.; Sanchiz, R. \& Grisolia, S. Growth, enzimes and hormonal changes in offspring of-fed ras, in mechanisms of alcohol damage in utero. Ciba Found. Symp., 105:85-102, 1984.

Guerri, C. \& Sanchis, R. Alcohol and acetaldehyde in rat's milk following ethanol administration. Life Sci., 38:1543$56,1986$.

Heil, H. H. \& Subramanian, M. G. Chronic alcohol exposure and lactation. Extended observations. Alcohol, 21:12732, 2000 .

Herrera, E. \& Freinkel, N. Interrelationships between liver composition, plasma glucose and ketones, and hepatic acetyl CoA and citric acid during prolonged starvation in the male rat. Biochim.Biophys. Acta, 170:244-53, 1968.

Jones, K. L.; Smith, D. W.: Ulleland, C. N. \& Streissguth, A.P. Pattern of malformations in offspring of chronic alcoholic mothers. Lancet, 1:1267-71, 1973.

Jones, K.L. \& Stewart, D.B. Effects of orally-administered ethanol on mammary gland morphology and functional efficiency in lactating rats. Exp.Pathol., 25:205-13, 1984.

LeMoine, P.; Harousseau, H.; Borteyru, J.P. \& Menuet, J.C. Les enfants de parents alcooliques: Anomalies observées à propos de 127 cas. Quest.Méd., 25:476, 1968.

Lieber, C.S. Interactions of alcohol and nutrition. Introduction to a Symposium. Alcohol Clin. Exp. Res., 7:2-4, 1983.

Little, R. E.; Anderson, K. W.; Erwin, C. H.; Worthington, R. B. \& Clarren, S. K. Maternal alcohol use during breastfeeding and infant mental and motor development at one year. N. Engl. J. Med., 321:425-30, 1989.

Ludeña, M. C.; Mena, M. A.; Salinas, M. \& Herrera, E. Effects of alcohol ingestion in the pregnant rat on daily food intake, offspring growth and metabolic parameters. Gen. Pharmac., 14:327-32, 1983.

Matsuda, Y.; Baraona, E.; Salaspuro, M. \& Lieber, C.S. Effects of ethanol on liver microtubules and Golgi apparatus. Lab. Invest., 41:455-63, 1979.

Menella, J. A. \& Beauchamp, G. K. Beer breast feeding, and folklore. Dev. Psychobiol., 26:459-66, 1993.
Oyama, L. M.; Couto, R. C.; Couto, G. E. C.; Dâmaso, A. R. \& Oller-do-Nascimento, C. M. Ethanol intake during lactation. I. Effects on dams' metabolism and pups' body weight gain. Alcohol, 21:195-200, 2000a.

Oyama, L. M.; Couto, R. C.; Couto, G. E. C.; Dâmaso, A. R. \& Oller-do-Nascimento, C.M. Ethanol intake during lactation. II. Effects on pups' liver and brain metabolism. Alcohol, 21:201-6, 2000b.

Rosett, H. L. Clinical pharmacology of the fetal alcohol syndrome. In: Majchrowics, E. \& Noble, E.P. (ed.), Biochemistry and Pharmacology of Ethanol. New York: Plenum Press, 1979. V. 2.

Sala, M. A.; Matheus, M. \& Valeri, V. A new stereological method for estimating the thickness of a cellular layer on random sections. Mikroskopie, 38: 127-30, 1981.

Sala, M.A.; Lopes, R.A. \& Matheus, M. Método morfológico para analisis cuantitativa de los tejidos. Determinacíon de los parâmetros normalies para el hepatocito de rata. Arch. Fac. Med. Zaragoza, 32:29-31, 1992.

Sala, M. A.; Komesu, M. C.; Lopes, R. A. \& Maia Campos, G. Karyometric study fo basal cell carcinoma. Braz. Dental J., 5:11-4, 1994.

Sanchis, R. \& Guerri, C. Chronic ethanol intake in lactating rats: Milk analysis. Comp. Biochem.Physiol., 85:107-10, 1986.

Shorey, R. L.; Terranella, P. A. \& Shive, W. Effects of ethanol on growth, consumption of food, and body composition of weanling rats. J. Nutr., 107:614-20, 1977.

Sprent, P. \& Smeeton, N. C. Applied nonparametric statistical methods. 3rd. ed. Boca Raton, Chapman \& Hall/CRC, 2001.

Steven, W. M.; Bulloch, B. \& Seelig Jr, L. L. A morphometric study of the effect of ethanol consumption on lactating mammary glands of rats. Alcohol. Clin. Exp. Res., 13: 209-12, 1989.

Subramanian, M. G. \& Abel, E.L. Alcohol inhibits sucklinginduced prolactin release and milk yield. Alcohol, 5: 958, 1988.

Subramanian, M. G. Evaluation of lactational parameters after alcohol administration for four days during early or midlactation in the rat. Alcohol Clin. Exp.Res., 21: 799-803, 1997. 
Tavares-do-Carmo, M.G.; Oller-do-Nascimento-Curi, C.M.; Martin-Hidalgo, A. \& Herrera, E. Effects of ethanol intake on lipid metabolism in the lactating rat. Alcohol, 13:443-8, 1996.

Tavares-do-Carmo, M.G.; Oller-do-Nascimento-Curi, C.M.; Martin, A. \& Herrera, E. Ethanol intake during lactation impairs milk production in rats and affects growth and metabolism of suckling pups. Alcohol, 18:71-6, 1999.

Testar, X.; Llobera, M. \& Herrera, E. Comparative metabolic effects of chronic ethanol intake and undernutrition in pregnant rat and their fetuses. Alcoholism Clin. Exp. Res., 12:197-200, 1988.

Testar, X.; López, D.; Llobera, M. \& Herrera, E. Ethanol administration in the drinking fluid to pregnant rats as a model for the fetal alcohol syndrome. Pharmacol. Biochem. Behav., 24:625-30, 1986.

Tomkeieff, S. I. Linear intercepts, areas and volumes. Nature, 155:24, 1945.

Vilaró, S.; Viñas, O.; Remesar, X. \& Herrera, E. Effects of chronic ethanol consumption on lactational performance in rat: Mammary gland and milk composition and pups' growth and metabolism. Pharmacol. Biochem. Behav., 27:333-9, 1987.

Vilaró, S.; Viñas, O. \& Remesar, X. Altered ultrastructure of lactating rat mammary epithelial cells induced by chronic ethanol ingestion. Alcohol. Clin. Exp. Res., 13:128-36, 1989.

Viñas, O.; Vilaró, S.; Herrera, E. \& Remesar, X. Effects of chronic ethanol treatment on amino acid uptake and enzyme activities in the lactating rat mammary gland. Life Sci., 40:1745-9, 1987.

Volpi, R.; Chiodera, P.; Gramellini, D.; Cigarini, C.; Papadia, C.; Caffarri,G.; Rossi, G. \& Coiro, V. Endogenous opioid mediation of the inhibition effect of ethanol on the prolactin response to breast stimulation in normal women. Life Sci., 54:739-44, 1994.

Wallgren, H.; Ahlqvist, J.; Ahman, K. \& Suomalainen, H. Repeated alcoholic intoxication compared with continued consumption of dilute ethanol in experiments with rats on a marginal diet. Brit. J. Nutr., 21:643-60, 1967.
Dirección para correspondência:

Prof. Dr. Ruberval A. Lopes

Departamento de Morfologia, Estomatologia e Fisiologia

Faculdade de Odontologia de Ribeirão Preto

Universidade de Säo Paulo

Av. do Cafe, s/n

CEP14040-904

Ribeirão Preto, $S P$

BRASIL

Email: ruberlopes@hotmail.com

Recibido : 04-07-2005

Aceptado: 15-09-2005 